02

\title{
Новый метод определения распределения плотности электронных состояний в хвосте валентной зоны аморфных твердых растворов $\mathrm{Se}_{x} \mathrm{~S}_{1-x}$
}

\author{
(C) Р.Г. Икрамов, М.А. Нуриддинова, Х.А. Муминов
}

Наманганский инженерно-технологический институт, 160115 Наманган, Узбекистан

e-mail: rgikramov@mail.ru

Поступила в редакцию 01.03.2021 г.

В окончательной редакции 07.05.2021 г.

Принята к публикации 13.05.2021 г.

Сопоставлены аналитическое выражение и экспериментальная кривая спектра экспоненциального поглощения аморфного селено-серного твердого раствора $\left(\mathrm{Se}_{0.5} \mathrm{~S}_{0.5}\right.$ и $\left.\mathrm{Se}_{0.7} \mathrm{~S}_{0.3}\right)$. Параметры в аналитическом выражении спектра экспоненциального поглощения, определяющие кривизну экспоненциальных хвостов разрешенных зон, найдены путем аппроксимации экспериментальной кривой. Используя приближение Дэвиса-Мотта по формуле Кубо-Гринвуда для спектра экспоненциального поглощения получена новая формула, определяющая плотности электронных состояний в хвосте валентной зоны. С использованием этой формулы и экспериментально определенного спектра экспоненциального поглощения показана возможность определения плотности электронных состояний в хвосте валентной зоны.

Ключевые слова: аморфные полупроводники, хвосты разрешенных зон, формула Кубо-Гринвуда, приближение Дэвиса-Мотта, оптические электронные переходы, спектр экспоненциального поглощения, параметры, определяющие крутизну хвостов разрешенных зон, распределение плотности электронных состояний.

DOI: $10.21883 /$ OS.2021.11.51636.1949-21

Как известно, аналитический спектр оптического поглощения аморфных полупроводников вычисляют по методу Дэвиса-Мотта из формулы КубоГринвуда [1]:

$$
\alpha(\hbar \omega)=A \int g(\varepsilon) g(\varepsilon+\hbar \omega) \frac{d d \varepsilon}{\hbar \omega} .
$$

Здесь $A-$ не зависящий от частоты поглощенного фотона коэффициент пропорциальности, равный $A=\frac{8 \pi^{4} e^{2} \hbar^{2} a}{n c\left(m^{*}\right)^{*}}$, где $e-$ заряд электрона, $a-$ среднее растояние между атомами аморфного полупроводника, $n$ - показатель преломления, $c$ - скорость света в вакууме, $m^{*}$ - эффективная масса электрона, $\hbar$ - постоянная Планка, $\omega$ - частота поглощенных фотонов, $g(\varepsilon)$ и $g(\varepsilon+\hbar \omega)$ - начальное и конечное плотности электронных состояний электронов, участвующих в оптических переходах соответственно.

Из формулы (1) видно, что спектр оптического поглощения зависит от распределения плотности электронных состояний, участвующих в оптических переходах. Поэтому в настоящей работе предлагается новый метод определения распределения плотности электронных состояний в хвосте валентной зоны из экспериментального спектра экспоненциального поглощения.
В области экспоненциального поглощения выполняется правило Урбаха и условия для энергии поглощенных фотонов $\varepsilon_{C}-\varepsilon_{V}=E_{g}>\hbar \omega$, где $E_{g}-$ энергетическая ширина щели подвижности [2]. Здесь $\varepsilon_{V}-$ потолок валентной зоны и $\varepsilon_{C}$ - дно зоны проводимости. При поглощении таких фотонов одновременно происходят следующие оптические переходы электронов: от хвоста валентной зоны в зону проводимости, от хвоста валентной зоны в хвост зоны проводимости и от валентной зоны в хвост зоны проводимости (рис. 1).

Интеграл в формуле (1) является неопределенным, поэтому из этой формулы можно получить только общее решение, но не собственное. Поэтому в работе [3] формула Кубо-Гринвуда написана определенным интегралом в следующем виде:

$$
\alpha(\hbar \omega)=A \int_{\varepsilon_{0}-\hbar \omega}^{\varepsilon_{0}} g(\varepsilon) g(\varepsilon+\hbar \omega) \frac{d \varepsilon}{\hbar \omega},
$$

где $\varepsilon_{0}-$ энергетическое положение точки пересечения экспоненциальных хвостов разрешенных зон (рис. 1). Эту формулу расчленили на парциальные коэффициенты поглощения, соответствующие указанным выше оптиче- 
ским переходам:

$$
\begin{aligned}
& \alpha(\hbar \omega)=A \int_{\varepsilon_{0}-\hbar \omega}^{\varepsilon_{0}} g(\varepsilon) g(\varepsilon+\hbar \omega) \frac{d \varepsilon}{\hbar \omega} \\
& =A \int_{\varepsilon_{C}-\hbar \omega}^{\varepsilon_{0}} g(\varepsilon) g(\varepsilon+\hbar \omega) \frac{d \varepsilon}{\hbar \omega} \\
& +A \int_{\varepsilon_{V}}^{\varepsilon_{C-\hbar \omega}} g(\varepsilon) g(\varepsilon+\hbar \omega) \frac{d \varepsilon}{\hbar \omega} \\
& +A \int_{\varepsilon_{0}-\hbar \omega}^{\varepsilon_{V}} g(\varepsilon) g(\varepsilon+\hbar \omega) \frac{d \varepsilon}{\hbar \omega} \\
& =\alpha_{1}(\hbar \omega)+\alpha_{2}(\hbar \omega)+\alpha_{3}(\hbar \omega) .
\end{aligned}
$$

Результаты расчетов значений этих парциальных коэффициентов поглощения показывают, что при определении коэффициента экспоненциального поглощения основную роль играют оптические электронные переходы от хвоста валентной зоны в хвост зоны проводимости, т. е. $\alpha_{1}(\hbar \omega)$ (рис. 2$)$.

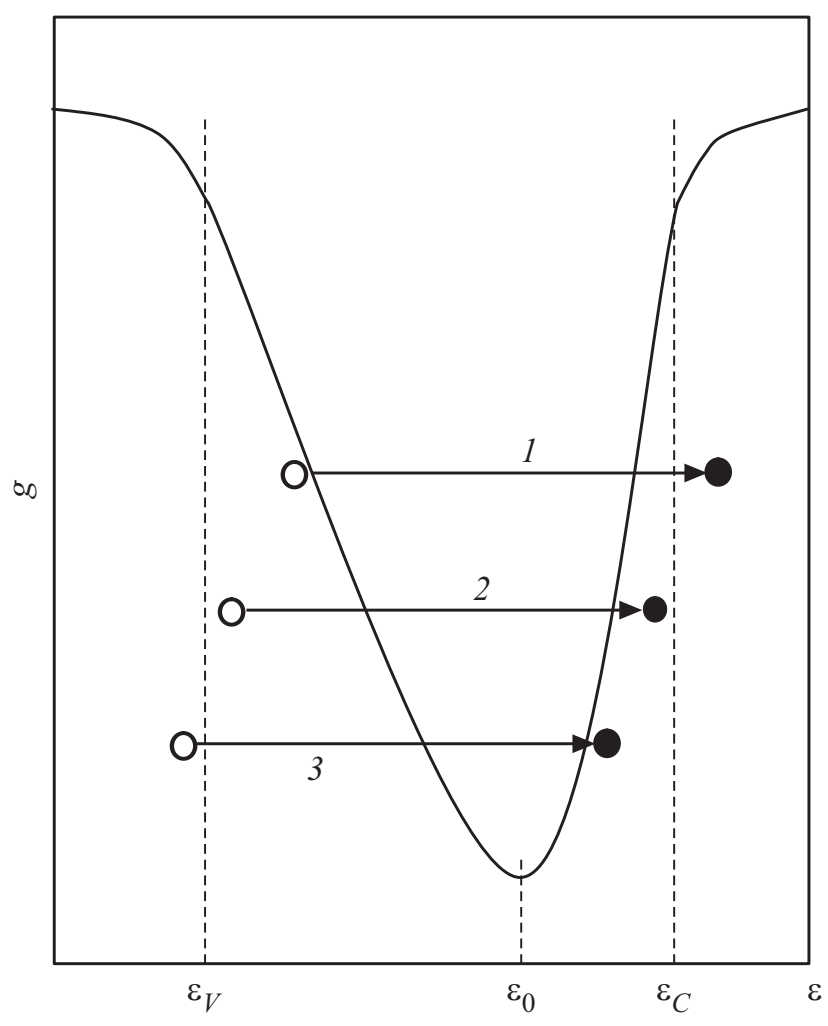

Рис. 1. Виды оптических переходов в аморфных полупроводниках, при которых энергия поглощенных фотонов лежит в интервале $\hbar \omega<\varepsilon_{c}-\varepsilon_{V}=E_{g}$ : от хвоста валентной зоны в зону проводимости (1), от хвоста валентной зоны в хвост зоны проводимости (2), от валентной зоны в хвост зоны проводимости (3).

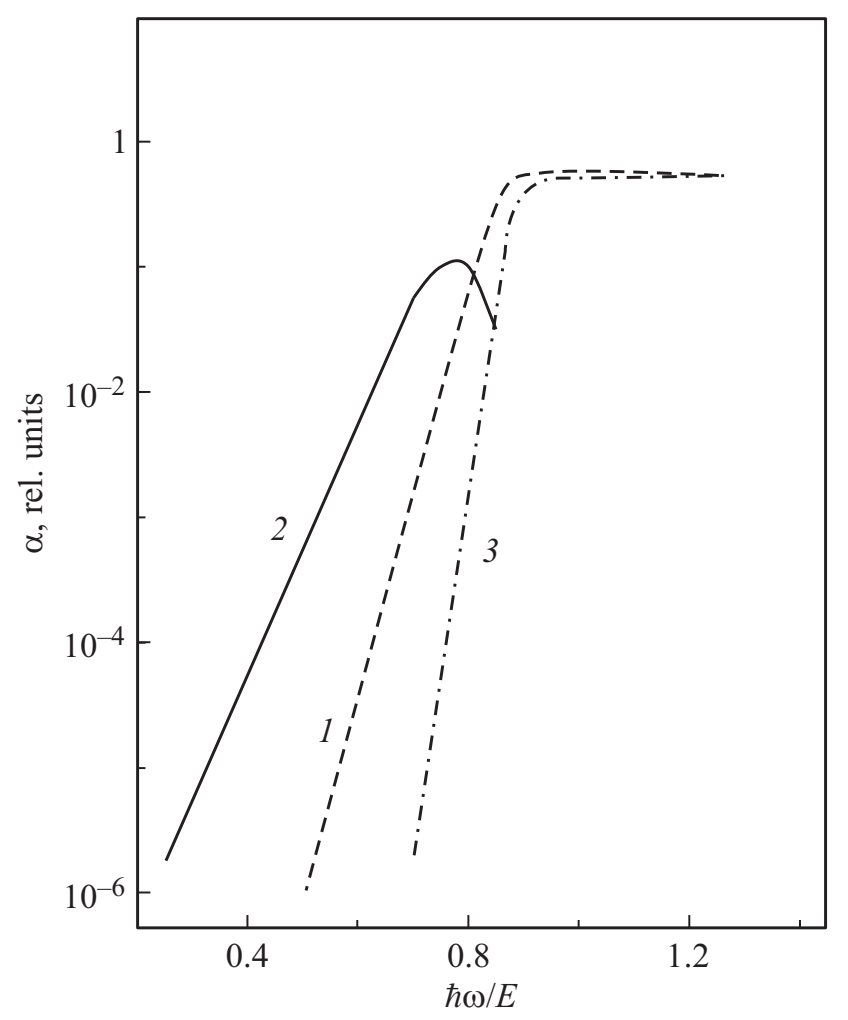

Рис. 2. Спектральные зависимости парциальных коэффициентов поглощения, соответствующих оптическим переходам электронов, при которых энергия поглощенных фотонов лежит в интервале $\hbar \omega<\varepsilon_{c}-\varepsilon_{V}=E_{g}: \alpha_{1}-$ от хвоста валентной зоны в зону проводимости $(1), \alpha_{2}-$ от хвоста валентной зоны в хвост зоны проводимости (2), $\alpha_{3}$ - от валентной зоны в хвост зоны проводимости (3) [3].

Поэтому в работе [4] для спектра коэффициента экспоненциального поглощения формула Кубо-Гринвуда в приближении Дэвиса-Мотта написана следующим образом:

$$
\alpha(\hbar \omega)=A \int_{\varepsilon_{V}}^{\varepsilon_{C}-\hbar \omega} g_{1}(\varepsilon) g_{2}(\varepsilon+\hbar \omega) \frac{d \varepsilon}{\hbar \omega},
$$

где $g_{1}(\varepsilon)$ - распределение плотности электронных состояний в хвосте валентной зоны, а $g_{2}(\varepsilon+\hbar \omega)-$ распределение плотности электронных состояний в хвосте зоны проводимости.

В работе [5] распределение плотностей электронных состояний в экспоненциальных хвостах разрещенных зон написано в виде

$$
g_{1}(\varepsilon)=N\left(\varepsilon_{V}\right) \exp \left(-\beta_{1}\left(\varepsilon-\varepsilon_{V}\right)\right)
$$

где $\varepsilon_{V}<\varepsilon<\varepsilon_{0}$, для хвоста валентной зоны и

$$
g_{2}(\varepsilon)=N\left(\varepsilon_{C}\right) \exp \left(\beta_{2}\left(\varepsilon-\varepsilon_{C}\right)\right),
$$

где $\varepsilon_{0}<\varepsilon<\varepsilon_{C}$ для хвоста зоны проводимости. 


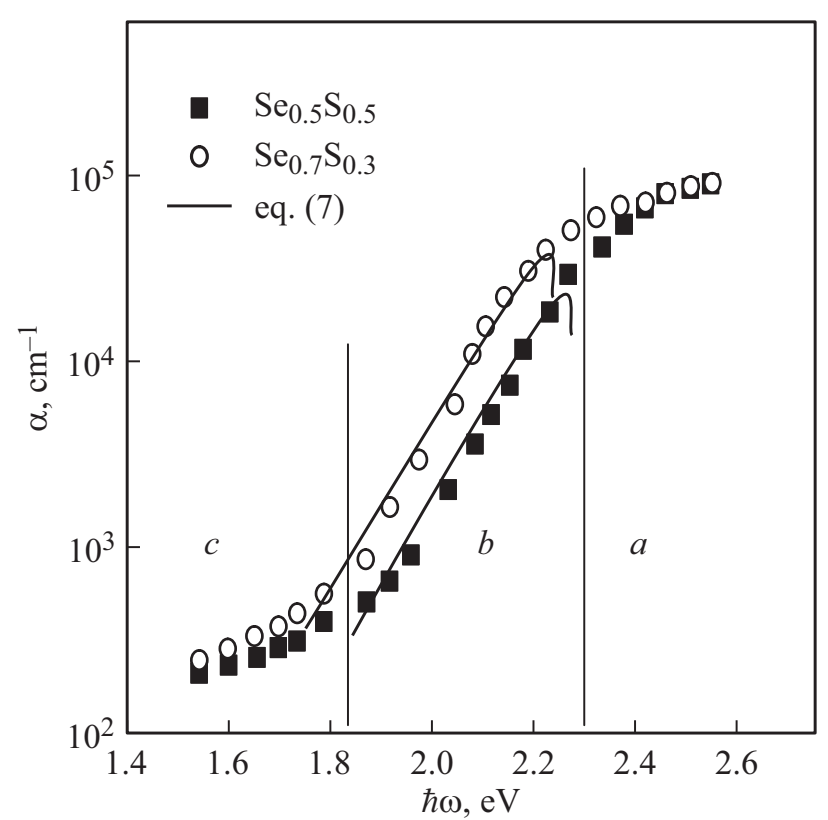

Pис. 3. Спектр, полученный для аморфных $\mathrm{Se}_{0.5} \mathrm{~S}_{0.5}$ и $\mathrm{Se}_{0.7} \mathrm{~S}_{0.3}$ из эксперимента (круги и квадраты) [8], а также по формуле (7) (сплошные линии): области межзонного $(a)$, экспонециального $(b)$, дефектного поглощения $(c)$.

В этих формулах $N\left(\varepsilon_{V}\right)$ и $N\left(\varepsilon_{C}\right)$ - эффективные значения плотности электронных состояний соответственно валентной зоны и зоны проводимости, $N\left(\varepsilon_{V}\right) \approx N\left(\varepsilon_{C}\right) \approx 10^{22} \mathrm{eV}^{-1} \mathrm{~cm}^{-3}[6], \beta_{1}$ и $\beta_{2}$ - параметры, определяющие кривизну экспоненциальных хвостов соответственно валентной зоны и зоны проводимости.

В работе [7] путём подстановки (5) и (6) в (4) для аналитического решения спектра экспоненциального поглощения получен следующий результат:

$$
\begin{aligned}
& \alpha(\hbar \omega)=\frac{B}{\left(\beta_{2}-\beta_{1}\right) \hbar \omega} \exp \left(\beta_{1}\left(\hbar \omega-E_{g}\right)\right) \\
& \times\left[1-\exp \left(\left(\beta_{2}-\beta_{1}\right)\left(\hbar \omega-E_{g}\right)\right)\right],
\end{aligned}
$$

где $B=A N\left(\varepsilon_{V}\right) N\left(\varepsilon_{C}\right)$.

Видно, что для определения значения аналитических решений спектра экспоненциального поглощения по формуле (7) необходимо определить постоянные $B, E_{g}$, $\beta_{1}$ и $\beta_{2}$. Для этого мы будем пользоваться результатами спектральных характеристик коэффициента экспоненциального поглощения, определенными экспериментально.

В работе [8] приведены экспериментальные результаты спектральных характеристик коэффициента оптического поглощения аморфного твердого раствора $\left(a-\mathrm{Se}_{x} \mathrm{~S}_{1-x}\right)$, полученного по методу термического напыления в вакууме. Для определения указанных выше постоянных мы выбираем результаты экспериментов, полученные для $a-\mathrm{Se}_{0.5} \mathrm{~S}_{0.5}$ и $a-\mathrm{Se}_{0.7} \mathrm{~S}_{0.3}$.

Эти спектры оптического поглощения разделяем на межзонные, экспоненциальные и дефектные области (рис. 3) [9].
В работе [10] значения $B$ и $E_{g}$ определены, рассматривая их в качестве подгоночных параметров, сравнивая результаты расчетов аналитического вида спектра межзонного поглощение, полученные для параболических разрешенных зон, с результатами экспериментов. Для $a-\mathrm{Se}_{0.5} \mathrm{~S}_{0.5} B=7.5 \cdot 10^{5} \mathrm{~cm}^{-1}$ и $E_{g}=2.25 \mathrm{eV}$, а для $a-\mathrm{Se}_{0.7} \mathrm{~S}_{0.3} \quad B=7.1 \cdot 10^{5} \mathrm{~cm}^{-1}$ и $E_{g}=2.2 \mathrm{eV}$. Для определения численных значений $\beta_{1}$ и $\beta_{2}$, рассматриваемых в качестве подгоночных параметров, результаты экспериментов для области экспоненциального поглощения сопоставлены с результатами расчетов по формуле (7) (рис. 3, сплошные линии). В результате получены значения $\beta_{1}=16.2 \mathrm{eV}^{-1}$ и $\beta_{2}=19.8 \mathrm{eV}^{-1}$ для $a-\mathrm{Se}_{0.5} \mathrm{~S}_{0.5}$, $\beta_{1}=16.3 \mathrm{eV}^{-1}$ и $\beta_{2}=19.7 \mathrm{eV}^{-1}$ для $a-\mathrm{Se}_{0.7} \mathrm{~S}_{0.3}$.

B работе [11] для производной по одной переменной от интеграла функции двух переменных приведено следующее выражение:

$$
\begin{aligned}
& \frac{d}{d y} \int_{\alpha(y)}^{\beta(y)} f(x, y) d x=\int_{\alpha(y)}^{\beta(y)} \frac{\partial f(x, y)}{\partial y} d x \\
& +\frac{\partial \beta(y)}{\partial y} f(\beta(y), y)-\frac{\partial \alpha(y)}{\partial y} f(\alpha(y), y) .
\end{aligned}
$$

Подставляя в эту формулу (2), получим следующее выражение:

$$
\begin{aligned}
& \frac{\partial \alpha(\hbar \omega)}{\partial \hbar \omega}=\frac{\partial}{\partial \hbar \omega} A\left(\int_{\varepsilon_{V}}^{\varepsilon_{C}-\hbar \omega}\left(\frac{g_{1}(\varepsilon) g_{2}(\varepsilon+\hbar \omega)}{\hbar \omega}\right) d \varepsilon\right) \\
& =\frac{A}{\hbar \omega} \int_{\varepsilon_{C}-\hbar \omega}^{\varepsilon_{V}} g_{1}(\varepsilon) \frac{\partial}{\partial \hbar \omega} g_{2}(\varepsilon+\hbar \omega) d \varepsilon \\
& -\frac{\alpha(\hbar \omega)}{\hbar \omega}-\frac{A}{\hbar \omega} g_{1}\left(\varepsilon_{C}-\hbar \omega\right) g_{2}\left(\varepsilon_{C}\right) .
\end{aligned}
$$

Подставив в эту формулу (3) и (4), получим

$$
\begin{aligned}
& \frac{\partial \alpha(\hbar \omega)}{\partial \hbar \omega}=\frac{A}{\hbar \omega} \int_{\varepsilon_{C}-\hbar \omega}^{\varepsilon_{v}} N\left(\varepsilon_{V}\right) N\left(\varepsilon_{C}\right) \exp \left(\beta_{1} \varepsilon_{V}\right) \\
& \times \exp \left(-\beta_{1} \varepsilon\right) \beta_{2} \exp \left(\beta_{2}\left(\varepsilon-\varepsilon_{C}+\hbar \omega\right)\right) d \varepsilon-\frac{\alpha(\hbar \omega)}{\hbar \omega} \\
& -\frac{A}{\hbar \omega} N\left(\varepsilon_{V}\right) N\left(\varepsilon_{C}\right) \exp \left(-\beta_{1}\left(E_{g}-\hbar \omega\right)\right) \\
& =\frac{A \beta_{2}}{\hbar \omega\left(\beta_{2}-\beta_{1}\right)} \exp \left(\beta_{2}\left(\hbar \omega-E_{g}\right)\right)-\frac{A}{\hbar \omega} \\
& \times \exp \left(-\beta_{1}\left(E_{g}-\hbar \omega\right)\right)\left(\frac{\beta_{2}}{\left(\beta_{2}-\beta_{1}\right)}+1\right)-\frac{\alpha(\hbar \omega)}{\hbar \omega} .
\end{aligned}
$$

В выражении (10) обозначаем

$$
\begin{aligned}
g_{1}(\varepsilon) & =N\left(\varepsilon_{V}\right) \exp \left(-\beta_{1}(\varepsilon-\varepsilon)_{V}\right) \\
& =N\left(\varepsilon_{V}\right) \exp \left(-\beta_{1}\left(E_{g}-\hbar \omega\right)\right) .
\end{aligned}
$$



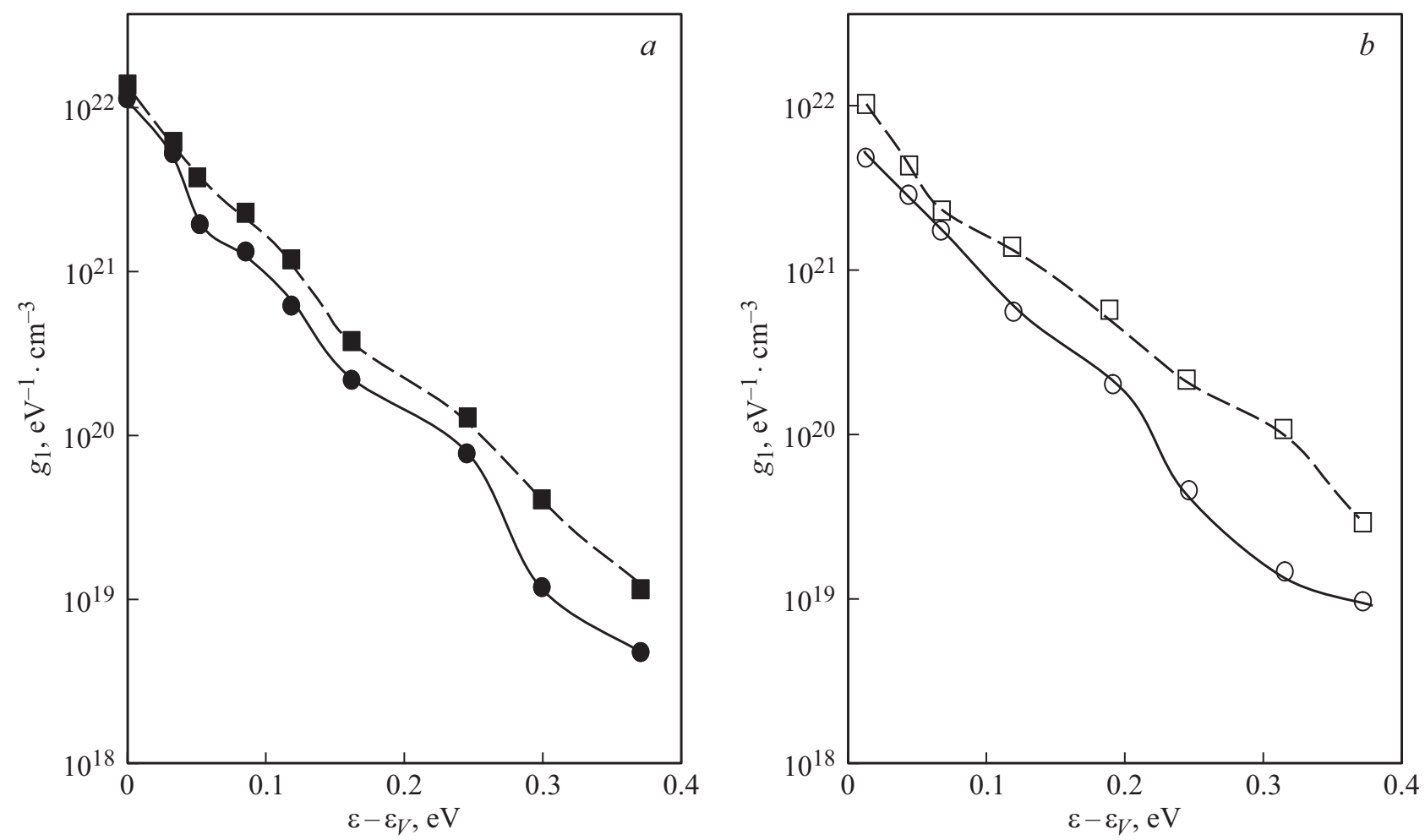

Рис. 4. Распределения плотности электронных состояний в хвосте валентной зоны для аморфных $\mathrm{Se}_{0.5} \mathrm{~S}_{0.5}(a)$ и $\mathrm{Se}_{0.7} \mathrm{~S}_{0.3}(b)$, рассчитанные по формулам (15) и (16) - соответственно сплошные (круги) и штриховые кривые (квадраты).

Используя (11), рассчитаем

$$
\varepsilon-\varepsilon_{V}=E_{g}-\hbar \omega, \quad \varepsilon=\varepsilon_{V}+E_{g}-\hbar \omega
$$

Поскольку в этом выражении $E_{g}>\hbar \omega, \varepsilon_{V}<0$, в формуле $(10) \varepsilon$ определяет энергетическое положение на хвосте валентной зоны. Поэтому из равенства (10) получим следующее выражение:

$$
\begin{aligned}
& \frac{\partial \alpha(\hbar \omega)}{\partial \hbar \omega}=\frac{B \beta_{2}}{\hbar \omega\left(\beta_{2}-\beta_{1}\right)} \exp \left(\beta_{2}\left(\hbar \omega-E_{g}\right)\right) \\
& -\frac{B}{\hbar \omega N\left(\varepsilon_{V}\right)} g_{1}(\varepsilon)\left(\frac{\beta_{2}}{\left(\beta_{2}-\beta_{1}\right)}+1\right)-\frac{\alpha(\hbar \omega)}{\hbar \omega}
\end{aligned}
$$

и далее

$$
g_{1}(\varepsilon)=\frac{\hbar \omega N\left(\varepsilon_{V}\right)\left(\frac{B \beta_{2}}{\hbar \omega\left(\beta_{2}-\beta_{1}\right)} \exp \left(\beta_{2}\left(\hbar \omega-E_{g}\right)\right)\right)-\frac{\partial \alpha(\hbar \omega)}{\partial \hbar \omega}-\frac{\alpha(\hbar \omega)}{\hbar \omega}}{B\left(\frac{\beta_{2}}{\left(\beta_{2}-\beta_{1}\right)}+1\right)} .
$$

В формуле (14) $\frac{\partial \alpha(\hbar \omega)}{\partial \hbar \omega} \approx \frac{\Delta \alpha(\hbar \omega)}{\Delta \hbar \omega}=\frac{\alpha\left(\hbar \omega_{i+1}\right)-\alpha\left(\hbar \omega_{i}\right)}{\hbar \omega_{i+1}-\hbar \omega_{i}}$. С использованием средних значений коэффициента экспоненциального поглощения и энергии поглощенных фо- тонов, формулу (14) запишем в виде

$$
\begin{aligned}
& g_{1}\left(\varepsilon_{i}\right)= \\
& =\frac{\left(\hbar \omega_{i+1}+\hbar \omega_{i}\right) N\left(\varepsilon_{V}\right) \times}{\times\left(\frac{2 B \beta_{2}}{\left(\hbar \omega_{i+1}+\hbar \omega_{i}\right)\left(\beta_{2}-\beta_{1}\right)} \exp \left(\beta_{2}\left(\frac{\left(\hbar \omega_{i+1}+\hbar \omega_{i}\right)}{2}-E_{g}\right)\right)\right)} \\
& -\frac{\left(\hbar \omega_{i+1}+\hbar \omega_{i}\right) N\left(\varepsilon_{V}\right)\left(\frac{\alpha\left(\hbar \omega_{i+1}\right)-\alpha_{i}\left(\hbar \omega_{i}\right)}{\hbar \omega_{i+1}-\hbar \omega_{i}}+\frac{\alpha\left(\hbar \omega_{i+1}\right)+\alpha_{i}\left(\hbar \omega_{i}\right)}{\hbar \omega_{i+1}+\hbar \omega_{i}}\right)}{2 B\left(\frac{\beta_{2}}{\left(\beta_{2}-\beta_{1}\right)}+1\right)}, \\
& g_{1}\left(\varepsilon_{i}\right)=N\left(\varepsilon_{V}\right) \exp \left(-\beta_{1}\left(\frac{\hbar \omega_{i}+\hbar \omega_{i+1}}{2}\right)\right) .
\end{aligned}
$$

Здесь $\alpha\left(\hbar \omega_{i}\right)$ и $\hbar \omega_{i}-$ соответственно коэффициент поглощения и энергия поглощенных фотонов, определенные экспериментально.

Поставляя в (15) и (16) результаты измерения спектра экспоненциального поглощения, рассчитаем распределения плотности электронных состояний в хвосте валентной зоны, рассчитанные для $a-\mathrm{Se}_{0.5} \mathrm{~S}_{0.5}$ (рис. $4, a$ ) и $a-\mathrm{Se}_{0.7} \mathrm{~S}_{0.3}$ (рис. 4, $b$ ) по формулам (15) и (16). Они почти повторяют друг друга. Видно, что значения плотности электронных состояний в хвосте валентной зоны увеличиваются с увеличением содержания селена в твердом растворе $a-\mathrm{Se}_{x} \mathrm{~S}_{1-x}$. 
Таким образом, в настоящей работе путем сравнения эспериментальных результатов с рассчитанными по аналитическому выражению для спектра экспоненциального поглощения показана возможность определения параметров, определяющих кривизну экспоненциальных хвостов валентной зоны и зоны проводимости. С использованием формулы Кубо-Гринвуда в приближении Дэвиса-Мотта для области экспоненциального поглощения выведена новая формула, определяющая распределения плотностей электронных состояний в хвосте валентной зоны. Применяя экспериментальные результаты, полученные для спектра экспоненциального поглощения, показана возможность определения распределения плотности электронных состояний в хвосте валентной зоны. Показано, что значения распределения плотности электронных состояний в хвосте валентной зоны увеличиваются с увеличением селена в твердом растворе $a-\mathrm{Se}_{x} \mathrm{~S}_{1-x}$.

\section{Список литературы}

[1] Taus J. The Optical Properties of Solid / Ed. by Abeles F. Amsterdam: North-Holland, 1972. 1026 p.

[2] Zaynobidinov S., Ikramov R.G., Nuritdinova M.A., Zhalalov R.M. // Ukr. J. Phys. 2008. V. 53. N 12. P. 1177.

[3] Zaynobidinov S., Ikramov R.G., Nuritdinova M.A., Zhalalov R.M. // Ukr. J. Phys. 2008. V. 53. N 8. P. 789.

[4] Зайнобидинов С., Икрамов Р.Г., Жалалов Р.М. // Журн. прикл. спектр. 2011. Т. 78. № 2. С. 243.

[5] Андреев А.А., Шлимак М.С. Фотоприемники и фотопреобразователи. Сб. науч. Тр. АН СССР. ФТИ им. Иоффе, Л.: Наука, 1986. 222 с.

[6] Cody G.D. // J. Non-Cryst. Solids. 1992. V. 141. P. 3.

[7] Зайнобидинов С., Икрамов Р.Г., Жалалов Р.М., Нуритдинова М.А. // Опт. и спектр. 2011. Т. 110. № 5. С. 813.

[8] Джсалилов Н.З., Дамиров Г.М. // ФТП. 2011. Т. 45. В. 4. C. 500 .

[9] Davis E.A., Mott N.F. Electronic Processes in Non Crystalline Materials. Oxford: Clarendon Press, 1979. 590 p.

[10] Ikramov R.G., Nuriddinova M.A., Muminov X.A. // J. Appl. Phys. Sci. International (JAPSI). 2020. V. 12. N 1. P. 36.

[11] Бронштейн И.Н., Семендяев К.А. Справочник по математике для инженеров и учащихся втузов. М.: Наука, Гл. Ред. Физ. Мат. Лит. 1986. 544 с. 